\title{
Raman-assisted BOTDA sensors
}

\author{
J. D. Ania-Castañón ${ }^{1}$, S. Martín-López ${ }^{1}$, M. Alcón-Camas ${ }^{2}$, F. Rodríguez-Barrios ${ }^{1}$, A. Carrasco-Sanz ${ }^{3}$, P. \\ Corredera $^{1}$, L. Thévenaz ${ }^{4}$ and M. González-Herráez ${ }^{5}$ \\ ${ }^{1}$ Inst. de Óptica, CSIC, Serrano 121, 28006, Madrid (Spain) \\ ${ }^{2}$ Photonics Research Group, Aston University, Birmingham B4 7ET, (United Kingdom) \\ ${ }^{3}$ Dept. de Óptica, Fac. de Ciencias, UGR, C. Universitario Fuentenueva, 18071, Granada (Spain) \\ ${ }^{4}$ Inst. of Elec. Engineering, EPFL. STI-GR-SCI Station 11, CH-1015, Lausanne (Switzerland) \\ ${ }^{5}$ Dept. de Electrónica, Universidad de Alcala, C. Universitario, 28871, Madrid (Spain) \\ Abstract: We review some recent results on the application of distributed Raman amplification schemes, including \\ ultralong lasers, to the extension of the operating range and contrast in Brillouin optical time domain analysis \\ (BOTDA) distributed sensing systems.
}

Brillouin optical time domain analysis (BOTDA) is a widely-used technique for the distributed measurement along optical fibers. The main interest of BOTDA lies in the possibility of using it to develop sensors for the continuous monitoring of temperature and/or strain in optical fibers [1-5]. Additionally, BOTDA has been used to perform power distribution measurements along optical fibers, enabling the measurement of fiber attenuation [6], chromatic dispersion [7] and parametric amplification [8]. The measurement range of BOTDA systems is typically shorter than $50 \mathrm{~km}$ [9] and generally limited to 20-30 km with a spatial resolution between 1-2 meters. The measurement range limitation is basically due to fiber attenuation. The losses in the fiber cause a drop of signal contrast with distance and a growth in the measurement uncertainty, as a simple result of the pump power reduction that critically scales the actual Brillouin gain. In these systems there is also a trade-off between resolution and measurement range: while it is desirable to have the highest possible resolution, this requires the use of short optical pulses, so the effective distance for amplification is reduced accordingly. This increases the difficulty in detection because the signal-to-noise ratio (SNR) is reduced. To some extent, one can compensate this by raising the pump power. However, the pump power cannot be increased indefinitely since other competing nonlinear effects (modulation instability and Raman) and also significant pump depletion start to take place. Thus, depending on system requirements, one has to choose between short pulses for increased resolution, or longer pulses for increased range. Several studies have been realized to extend the system resolution without impairing the measurement range. These rely on signal processing methods [10,11], distributed amplification along the fiber to overcome the fiber loss [12-14], or a combination of both [15]. In this talk we will review some of our recent results in the application of Raman-amplified schemes, including advanced higher-order amplification based on the ultralong laser architecture [16], to the improvement of the performance of BOTDA distributed sensors.

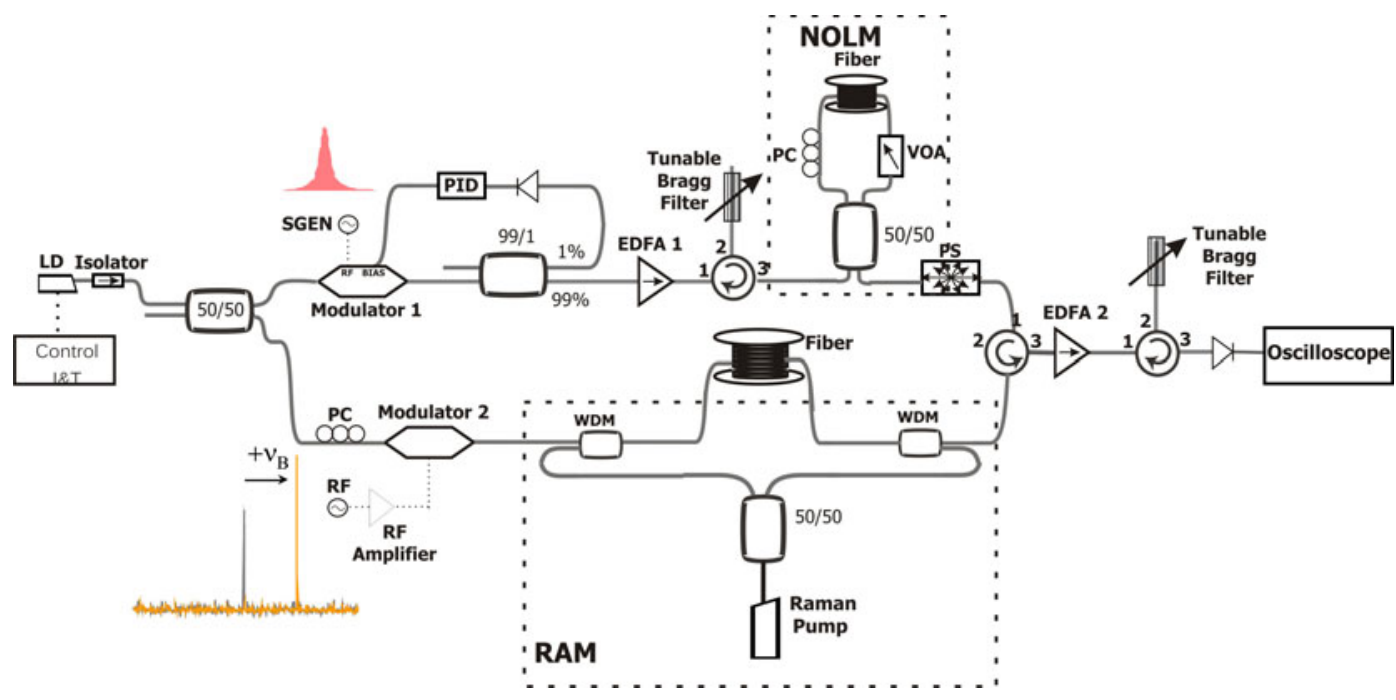

Figure 1 -Experimental setup of a Raman-assisted BOTDA. LD: Laser Diode; PC: Polarization controller; SGEN: Signal generator; PID: Proportional-Integral-Derivative electronic circuit; EDFA: Erbium Doped Fiber Amplifier. RF: Radio-frequency generator; PS: Polarization Scrambler; WDM: Wavelength Division Multiplexer; RAM: Raman Amplification Module. 
Fig. 1 shows a schematic diagram of the experimental setup used for the tests, including a conventional BOTDA sensor working at $1550 \mathrm{~nm}$. BOTDA systems use two different counter-propagating single-frequency light beams in the fiber. One of these light beams (centered at $f_{0}$ ) is pulsed and is meant to generate some amplification on the probe; we call this signal pump wave. The other one (centered around $f_{0}-v_{B}$, where $v_{B} \sim 10.5 \mathrm{Ho} 10.8 \mathrm{GHz}$ ) is a continuous wave, referred to as the probe wave, that will be locally amplified by the pump pulse through SBS. As the pump pulse travels down the fiber, it will induce a different amplification on the probe depending on the Brillouin shift. The local amplification of the probe wave will yield a time-dependent variation of the detected probe signal at the pump end. The amplification at each point will be maximized when the pump-probe frequency separation is exactly the Brillouin shift at that point. By scanning for this maximum at all the positions, one can obtain a map of the Brillouin shift of the fiber across its whole length. To improve the dynamic range of the BOTDA we introduce Raman pumping on one or both sides of the sensing fiber. Depending on whether we are using first-order pumping or an ultra-long Raman laser architecture, the Raman pump will operate at $1455 \mathrm{~nm}$ or at $1365 \mathrm{~nm}$. In the latter case, the sensing fiber will also incorporate two fiber Bragg gratings to create a resonant cavity.

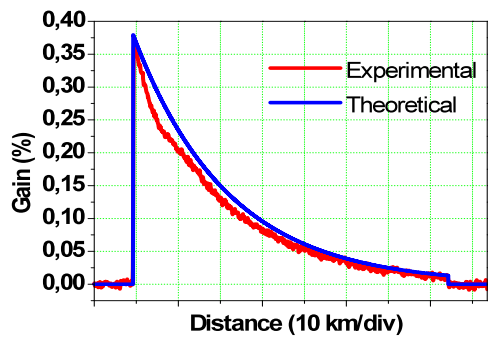

(a)

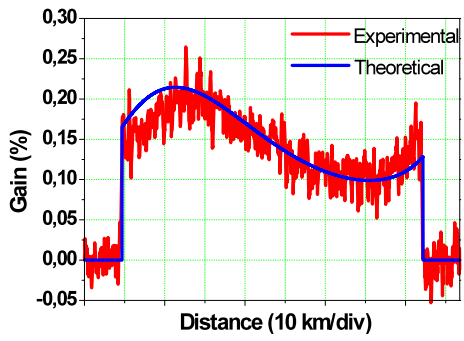

(b)

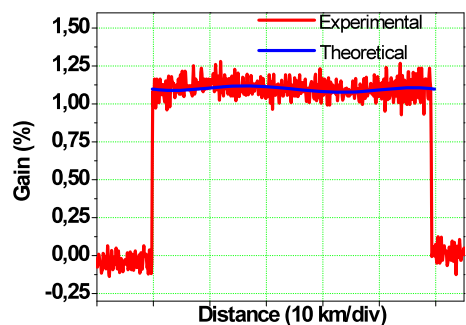

(c)

Fig.2. Theoretical and experimental gain traces obtained with the BOTDA: (a) without Raman amplification, (b) with bi-

directional Raman amplification and (c) with second-order bidirectional amplification provided by an ultralong laser, all with a

$2 \mathrm{~m}$ resolution

Figure 2 displays some sample results obtained with different amplification options, including no amplification at all, first-order bidirectional amplification and second-order ultralong laser amplification, in which the contrast improvement provided by the amplified solutions is clearly observed for distances of $75 \mathrm{~km}(\mathrm{a}$ and b) and $50 \mathrm{~km}$ (c). As we will show, although ultralong laser amplification has important advantages in terms of keeping contrast constant across the whole sensing line, there are some issues to address in order for this approach to be the most effective, the most important of which is keeping RIN transfer from the Raman pumps from affecting the quality of the BOTDA signals.

In conclusion, Raman amplification has proven itself an excellent method to improve the performance of distributed sensors based on stimulated Brillouin scattering for sensing temperature and strain without the need to resort to signal processing. Our experiments show that careful optimization of power ratios of both BOTDA signals and Raman pumps can allow close to constant contrast over up to $75 \mathrm{~km}$ with a $2 \mathrm{~m}$ resolution using only first-order amplification, while higher-order amplification could potentially allow us to extend measurement ranges well beyond the $100 \mathrm{~km}$.

1. T. Horiguchi, T. Kurashima and M. Tateda, IEEE Photon. Technol. Lett. 2, 352-354 (1990).

2. X. Bao, D. J. Webb, and D. A. Jackson, Opt. Lett. 18, 1561-1563 (1993)

3. M. Niklès, L. Thévenaz, and P. A. Robert, Opt. Lett. 21, 758-760 (1996)

4. M. DeMerchant, A. Brown, X.Bao, and T. Bremner, Appl. Opt. 38, 2755-2759 (1999)

5. H. Naruse, M. Tateda, H. Ohno and A. Shimada, Appl. Opt. 41 7212-7217 (2002).

6. T. Horiguchi and M. Tateda, Opt. Lett. 14, 408-410 (1989)

7. K.-Y. Song, M. González Herráez, and L. Thévenaz, J. Lightwave Technol. 23, 4140- (2005)

8. A. Vedadi, D. Alasia, E. Lantz, H. Maillotte, L. Thévenaz, M. González-Herráez, and T. Sylvestre, IEEE Photon. Technol. Lett. 19, 179181 (2007)

9. M. Niklès, Third European Workshop on Optical Fiber Sensors, 66190D, Italy, 2007.

10. A. W. Brown, M. D. DeMerchant, X. Bao, and T. W. Bremner, J. Lightwave Technol. 17, 1179-1183 (1999)

11. M. A. Soto, G. Bolognini, and F. Di Pasquale, Opt. Express 16, 19097-19111 (2008)

12. M. N. Alahbabi, Y. T. Cho, and T. P. Newson, J. Opt. Soc. Am. B 22, 1321-1324 (2005)

13. F. Rodriguez-Barrios, S. Martín-López, A. Carrasco-Sanz, P. Corredera, J. D. Ania-Castañón, L. Thévenaz and M. González-Herráez, J. Lightwave Technol. 28(15), 2162-2172 (2010)

14. Sonia Martin-Lopez, Mercedes Alcon-Camas, Felix Rodriguez, Pedro Corredera, Juan Diego Ania-Castañon, Luc Thévenaz, and Miguel Gonzalez-Herraez, Opt. Express 18, 18769-18778 (2010)

15. G. Bolognini and M. A. Soto, Opt. Express 18, 8459-8465 (2010)

16. J. D. Ania-Castañón, Opt. Express 12, 4372-4377 (2004) 